\title{
Variáveis Meteorológicas em Áreas de Floresta e Pastagem na Amazônia Ocidental em Anos de Eventos Extremos
}

Meteorological Variables in Forest and Pasture Area in the Western Amazon in years of extreme events

\section{Jayne Soares Martins do Nascimento ${ }^{1}$, Renata Gonçalves Aguiar ${ }^{2}$, Alberto Dresch Webler ${ }^{3}$, Graciela Redies Fischer ${ }^{4}$, Leonardo José Gonçalves Aguiar ${ }^{4}$ e Camila Bermond Ruezzene ${ }^{1}$}

\author{
${ }^{1}$ Graduanda em Engenharia Ambiental, Fundação Universidade Federal de Rondônia, \\ Ji-Paraná, Brasil \\ jayne_smn@hotmail.com; camila.ruezzene@gmail.com \\ ${ }^{2}$ Professora Doutora, Fundação Universidade Federal de Rondônia, Ji-Paraná, Brasil \\ rgaguiar@gmail.com \\ ${ }^{3}$ Professor Mestre, Fundação Universidade Federal de Rondônia, Ji-Paraná, Brasil \\ betowebler@gmail.com \\ ${ }^{4}$ Professor(a) Doutor(a), Universidade Federal de Pelotas, Pelotas, Brasil \\ graciela_fischer@yahoo.com.br; veraneiro@yahoo.com.br
}

\begin{abstract}
Resumo
O desmatamento na Amazônia é proveniente de contextos históricos que datam desde a colonização da região. Vários estudos já identificaram mudanças climáticas provenientes da conversão de florestas em sistemas agropecuários. Mediante o exposto, o presente estudo objetivou identificar possíveis diferenças no comportamento da umidade específica, temperatura do ar e do saldo de radiação em decorrência da conversão de floresta em pastagem, utilizando dados correspondentes aos anos de eventos extremos, visto que esses têm ocorrido em curto intervalo de tempo. Para tanto, foram utilizadas medidas das supracitadas variáveis de uma área de floresta tropical úmida e de uma área de pastagem, onde estão instaladas duas torres pertencentes ao Experimento de Grande Escala da Biosfera-Atmosfera na Amazônia - Programa LBA. As medições correspondem aos anos de 2005 e 2010, os quais ocorreram evento de seca, e 2009, um ano de cheia. Para cada variável foram calculadas as médias horárias de cada sítio, separadas por ano e por período. Verificou-se que os anos com eventos de seca proporcionaram maior variabilidade em todas as variáveis, principalmente no período seco dos anos citados, sendo a pastagem a área mais afetada. Entender essa dinâmica possibilita melhor planejamento das atividades agropecuárias da região.
\end{abstract}

Palavras-chave: Floresta Amazônica, mudanças climáticas, umidade específica, temperatura do ar, saldo de radiação.

\begin{abstract}
Deforestation in the Amazon comes from historical contexts which date from the colonization of the region. Several studies have identified climate change from the conversion of forests into agricultural systems. So, this study aimed to identify possible differences in the behavior of the specific humidity, air temperature and net radiation due to conversion of forest to pasture, using data corresponding to extreme event years, once these have occurred in short period of time. Therefore, it was used measures of the variables of a tropical rain forest area and a pasture area, where there are two towers belonging to the Large Scale Biosphere-Atmosphere Experiment in Amazonia-LBA. The measurements correspond to the years of 2005 and 2010, which occurred drought events, and 2009, a year that occurred a flood period. For each variable were calculated hourly means of each site, separated by year and by period. It was found that the years with dry events showed greater variability in all variables, especially in the dry period of the mentioned years, being the pasture the most affected area. Understanding this dynamic enables better planning in agricultural activities in the region.
\end{abstract}

Keywords: Amazon forest, climate change, specific humidity, air temperature, net radiation. 


\section{Introdução}

O desmatamento na Amazônia é proveniente de contextos históricos que datam desde a colonização da região, iniciada com o ciclo da borracha, até os dias atuais em que a predominância de atividades pecuárias é evidente (Soares-Filho et al., 2005). No entanto, o desmatamento foi intensificado em 1970, com a inauguração da rodovia Transamazônica (Fearnside, 2005).

As atividades pastoris causam a compactação do solo em grandes áreas, erosão, mudança no ciclo hidrológico e, consequentemente, queda na produtividade e biodiversidade (Fearnside, 2005). Além disso, a Amazônia possui papel fundamental no equilíbrio climático e, por isso, o aumento das áreas de pastagens, e consequentemente a redução da floresta, pode implicar em alterações na precipitação, umidade e temperatura do ar, radiação solar e na dinâmica do carbono, de forma a contribuir com o aquecimento global (Nobre; Sampaio; Salazar, 2007).

Modelos computacionais mostram que a derrubada de árvores na Floresta Amazônica pode ocasionar um clima cada vez mais quente e seco, podendo atingir uma escala em que esse local torne-se inapropriado para uma vegetação de grande porte, levando à perda total da floresta (Fearnside, 2009).

Estudo realizado por Nobre, Seller e Shukla (1991) há mais de duas décadas, evidenciou resultados importantes sobre a conversão de floresta em áreas de pastagens. Os autores constataram que a conversão provocou uma queda de $15 \%$ no saldo de radiação, reduziu em $30 \%$ a evapotranspiração, reduziu entre 20 e $30 \%$ a precipitação e aumentou a temperatura do ar entre 1 e $3^{\circ} \mathrm{C}$.

Gomes (2011) também constatou mudanças no microclima ao realizar um estudo similar na Amazônia Ocidental. Nesse foram constatados diminuição de $23 \%$ no saldo de radiação, redução pluviométrica de $7,25 \%$ no período úmido e $10,35 \%$ no período seco e decréscimo de $20 \%$ na umidade específica do ar.

É de suma importância identificar como a conversão de florestas tropicais em sistemas pecuários na Amazônia Ocidental influencia no microclima, uma vez que a compreensão de tais mudanças possibilita um planejamento mais eficiente da expansão da pecuária e da agricultura no estado de Rondônia.

Para o presente estudo foram analisados os anos nos quais ocorreram eventos extremos na região, visto que esses fenômenos têm se intensificado nos últimos anos. De acordo com Marengo (2009), o estudo de valores divergentes da média se torna ainda mais importante, pois esses trazem sérias consequências socioeconômicas.

No caso da Amazônia, esses eventos têm ocorrido em intervalos de tempo relativamente curtos. A região experimentou uma drástica seca em 2005, um evento de cheia em 2009 e outro de seca em 2010 (Marengo et al., 2011a; Marengo et al., 2011b).

Dessa forma, o presente estudo objetivou identificar possíveis discrepâncias no comportamento diário do saldo de radiação, umidade específica e temperatura do ar devido à conversão da floresta em pastagem, utilizando dados correspondentes aos anos de eventos extremos.

\section{Material e Métodos}

Este estudo foi realizado em dois sítios experimentais no estado de Rondônia, uma área de floresta tropical úmida (Floresta Amazônica) e outra em uma área de pastagem, onde estão instaladas duas torres pertencentes ao Experimento de Grande Escala da BiosferaAtmosfera na Amazônia - Programa LBA, desde 1999.

A área de floresta tropical úmida está localizada na Reserva Biológica do Jaru (REBIO

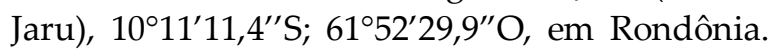
A REBIO Jaru é uma unidade de conservação federal de proteção integral do bioma amazônico, criada pelo Decreto 83.716, de 11 de julho de 1979, sob a tutela do Instituto Chico Mendes de Conservação da Biodiversidade (ICMBio).

A floresta é classificada como Floresta Ombrófila Aberta (Culf et al., 1997) e possui um rico sub-bosque de palmeiras de apenas alguns 
metros. O dossel tem altura média de aproximadamente $30 \mathrm{~m}$, mas algumas árvores emergentes chegam a medir $45 \mathrm{~m}$ (Rummel et al., 2002). O índice de área foliar (IAF) apresenta valores entre 5 e 6 (Andreae et al., 2002).

A Fazenda Nossa Senhora (FNS), 1045'44"S, $62^{\circ} 21^{\prime} 27^{\prime \prime} \mathrm{O}$, está situada no município de Ouro Preto do Oeste - Rondônia, $50 \mathrm{~km}$ a oeste de JiParaná (Figura 2) e dista aproximadamente 100 $\mathrm{km}$ da REBIO Jaru. A área foi desmatada no ano de 1977, com raio aproximado de $50 \mathrm{~km}$ e está situada na maior área plana com pastagem da região, a $220 \mathrm{~m}$ de altitude do nível do mar (CULF et al., 1997).

A cobertura vegetal predominante é a gramínea Brachiaria brizantha, além de pequenas palmeiras dispersas. O solo é classificado como podzólico vermelho amarelo (Hodnett et al., 1996). O fetch no sítio experimental é de aproximadamente $1-2 \mathrm{~km}$ em todas as direções (von Randow et al., 2004).

Foram utilizados dados de umidade relativa do ar (UR), temperatura do ar (Tar) e saldo de radiação $(\mathrm{Rn})$, dos anos correspondente a eventos extremos na região, sendo 2005 e 2010 anos de seca e 2009 um ano de cheia. Para o mesmo período foi calculado a umidade específica. Para as medições foi utilizado o termohigrômetro HMP35A e o HMP45C (Vaisala Inc., FIN), instalado a $10 \mathrm{~m}$ de altura na FNS e a $62 \mathrm{~m}$ na REBIO Jaru. O saldo de radiação foi medido pelo NR Lite 1 (Kipp \& Zonen, Delft, NLD). Esses dados foram coletados em intervalos de 30 segundos, e realizadas médias a cada 10 minutos que foram armazenadas em um datalogger CR10X na FNS e CR23X na REBIO Jaru, fabricados pela Campbell Scientific Instrument, Utah, USA.

Para cada variável foram calculadas médias horárias de cada sítio, separadas por ano e por período. Foram analisados os períodos úmido e seco, que correspondem aos meses de janeiro a março e julho a setembro, respectivamente.

A umidade específica do ar do período úmido dos anos de 2005 e 2010, assim como a temperatura do ar e o saldo de radiação do período úmido de 2005 não puderam ser analisados devido ao alto percentual de falhas nesses dados.

\section{Resultados e discussão}

\subsection{Umidade Específica do Ar}

Ao analisar a variação do ciclo diurno médio da umidade específica na REBIO Jaru e FNS (Figura 1), nota-se que a REBIO Jaru apresentou maior umidade específica, tanto no período úmido quanto no período seco, como também nos anos de seca (2005 e 2010) e de cheia (2009). Resultados já esperados devido a maior evapotranspiração em ecossistemas de floresta do que em ecossistemas de pastagem, bem como a maior disponibilidade hídrica da floresta, que possui um índice médio de chuvas $20 \%$ superior ao da pastagem.

É possível observar que para o período seco, durantes os anos de seca a umidade específica possui valores menores do que para o período úmido (em média $2 \mathrm{~g} \mathrm{~kg}^{-1}$ menor). Dessa forma, o período seco proporcionou menor umidade do ar tanto na floresta como na pastagem, como já evidenciado em trabalhos anteriores (Aguiar, 2006; Webler, 2013). No período úmido para o ano de 2009, os valores de umidade específica para a REBIO Jaru ficaram em torno de $18 \mathrm{~g} \mathrm{~kg}^{-1}$ e para a FNS ficaram próximos de $16 \mathrm{~g} \mathrm{~kg}^{-1}$.

Dentre os anos de seca, para o período seco, verifica-se que para a REBIO Jaru o ano de 2005 apresentou valores ligeiramente menores de umidade específica. E na FNS, 2010 apresentou os menores valores de umidade específica.

\subsection{Temperatura do Ar}

O ciclo diário da temperatura do ar é apresentado na Figura 2. Verifica-se que de um ano para outro, a diferença na temperatura do ar não foi tão expressiva, mas é perceptível que no período úmido a amplitude foi menor, tanto na floresta quanto na pastagem. Observando-se maiores valores de temperatura do ar no período seco, bem como menores temperaturas do ar. 
Período Úmido

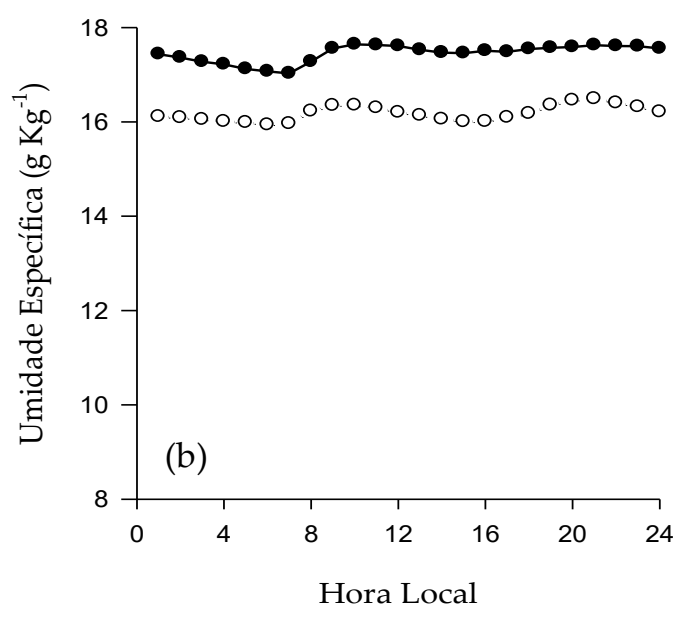

Período Seco
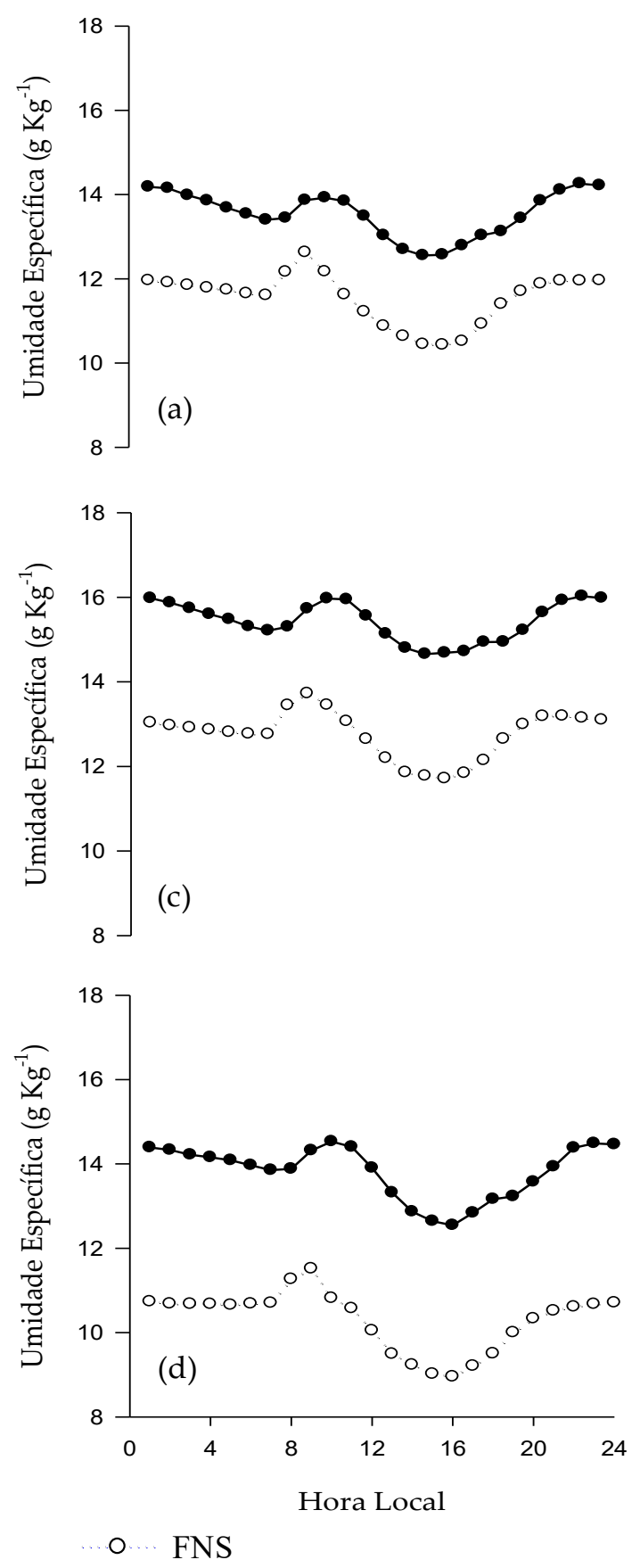

Figura 1: Variação do ciclo diurno médio da umidade específica do ar para o período úmido e seco da REBIO Jaru e FNS nos anos de 2005 (a), 2009 (b, c) e 2010 (d).

A amplitude térmica média é apresentada na Tabela 1. No período seco a amplitude térmica tende a ser maior, principalmente na pastagem. A umidade atua como controlador térmico, devido ao alto calor específico da água (Andrade et al., 2009), proporcionando temperaturas mais amenas e homogêneas no período úmido.
Ressalta-se que, nos anos com eventos de seca, a área de pastagem no período seco apresentou maior amplitude da temperatura quando comparada ao ano com evento de cheia. 

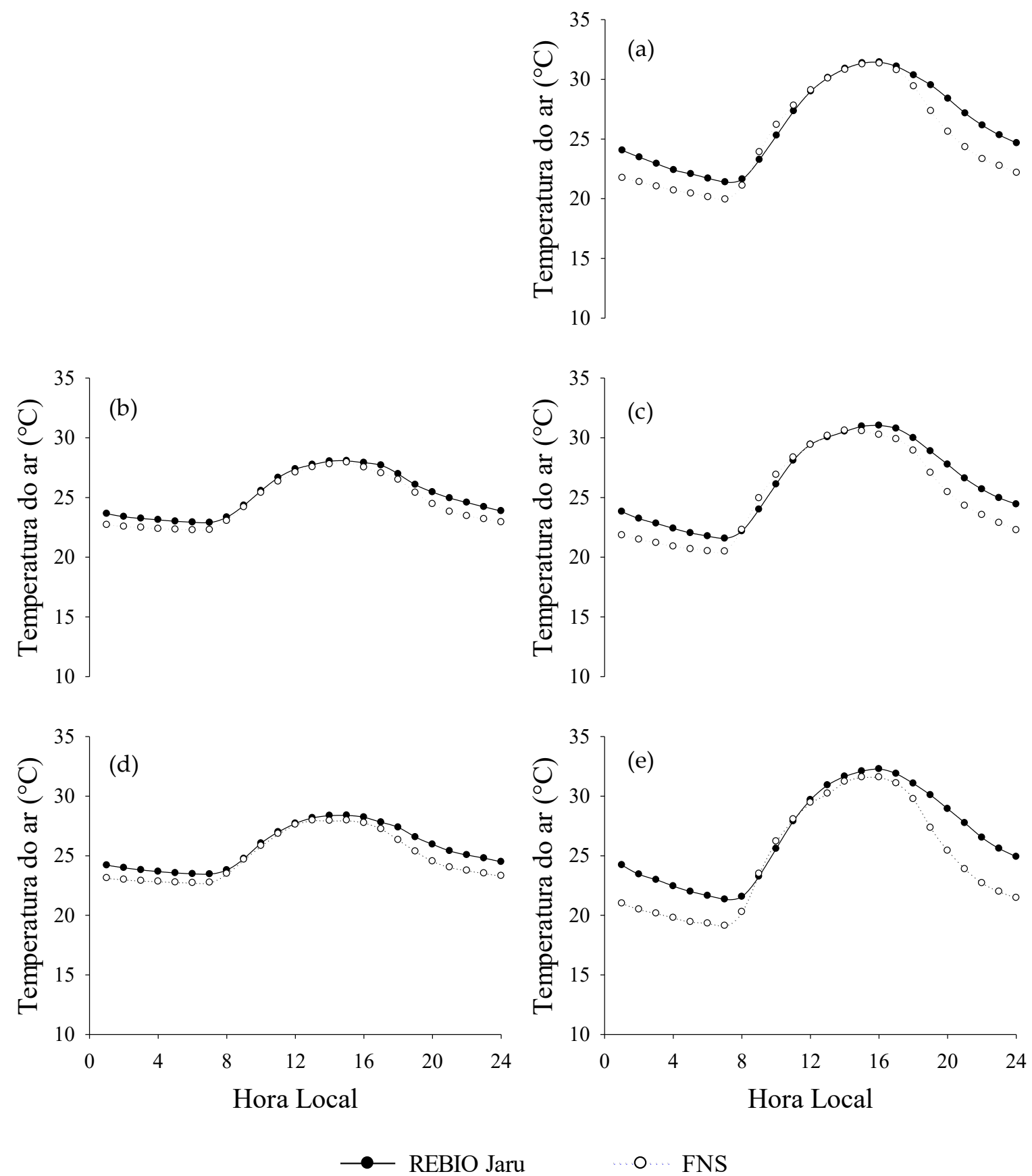

o.... FNS

Figura 2: Variação do ciclo diurno médio da temperatura do ar para o período úmido e seco da REBIO Jaru e FNS nos anos de 2005 (a), 2009 (b, c) e 2010 (d, e).

Tabela 1: Amplitude térmica média da temperatura do ar para os períodos úmido e seco da REBIO Jaru e FNS

\begin{tabular}{ccccc}
\hline \multicolumn{2}{c}{ REBIO Jaru } & \multicolumn{2}{c}{ FNS } \\
\hline & Úmido & Seco & Úmido & Seco \\
$\mathbf{2 0 0 5}$ & - & $10,93^{\circ} \mathrm{C}$ & $6,95^{\circ} \mathrm{C}$ & $12,41^{\circ} \mathrm{C}$ \\
$\mathbf{2 0 0 9}$ & $7,35^{\circ} \mathrm{C}$ & $10,67^{\circ} \mathrm{C}$ & $7,36^{\circ} \mathrm{C}$ & $11,59^{\circ} \mathrm{C}$ \\
$\mathbf{2 0 1 0}$ & $6,79^{\circ} \mathrm{C}$ & $11,89^{\circ} \mathrm{C}$ & $7,26^{\circ} \mathrm{C}$ & $13,38^{\circ} \mathrm{C}$ \\
\hline
\end{tabular}


No entanto, na área de floresta, embora haja uma variação da amplitude térmica, não foi possível definir um padrão em relação aos anos com eventos de cheia e de seca.

\subsection{Saldo de Radiação}

Pôde ser observado maiores valores de saldo de radiação na floresta tanto no período úmido

\section{Período Úmido}
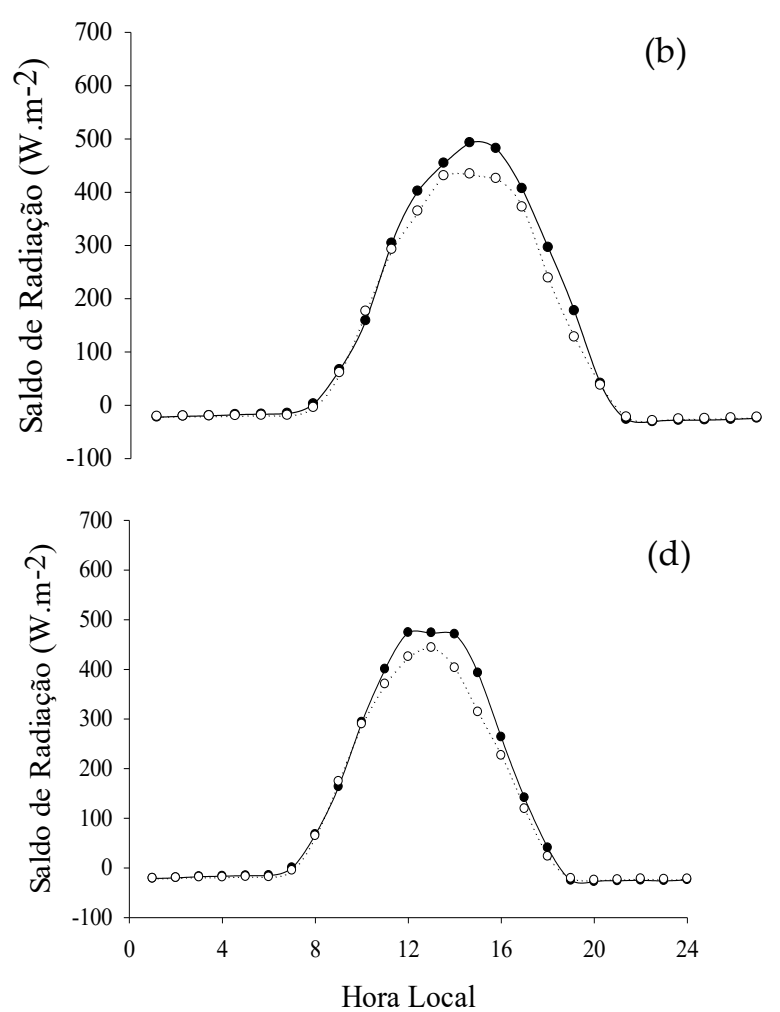

$\longrightarrow$ REBIO Jaru quanto no período seco, para todos os anos estudados (Figura 3).

Os anos em que ocorreram eventos de seca apresentaram menor saldo radiativo em relação a 2009, que teve um evento de cheia. A FNS apresentou a maior diferença de um ano para o outro, com queda de $12 \%$ no saldo de radiação dos anos 2005 e 2010 comparados a 2009, enquanto a REBIO Jaru teve diferença de 1,5\% em 2005 e 8\% em 2010.
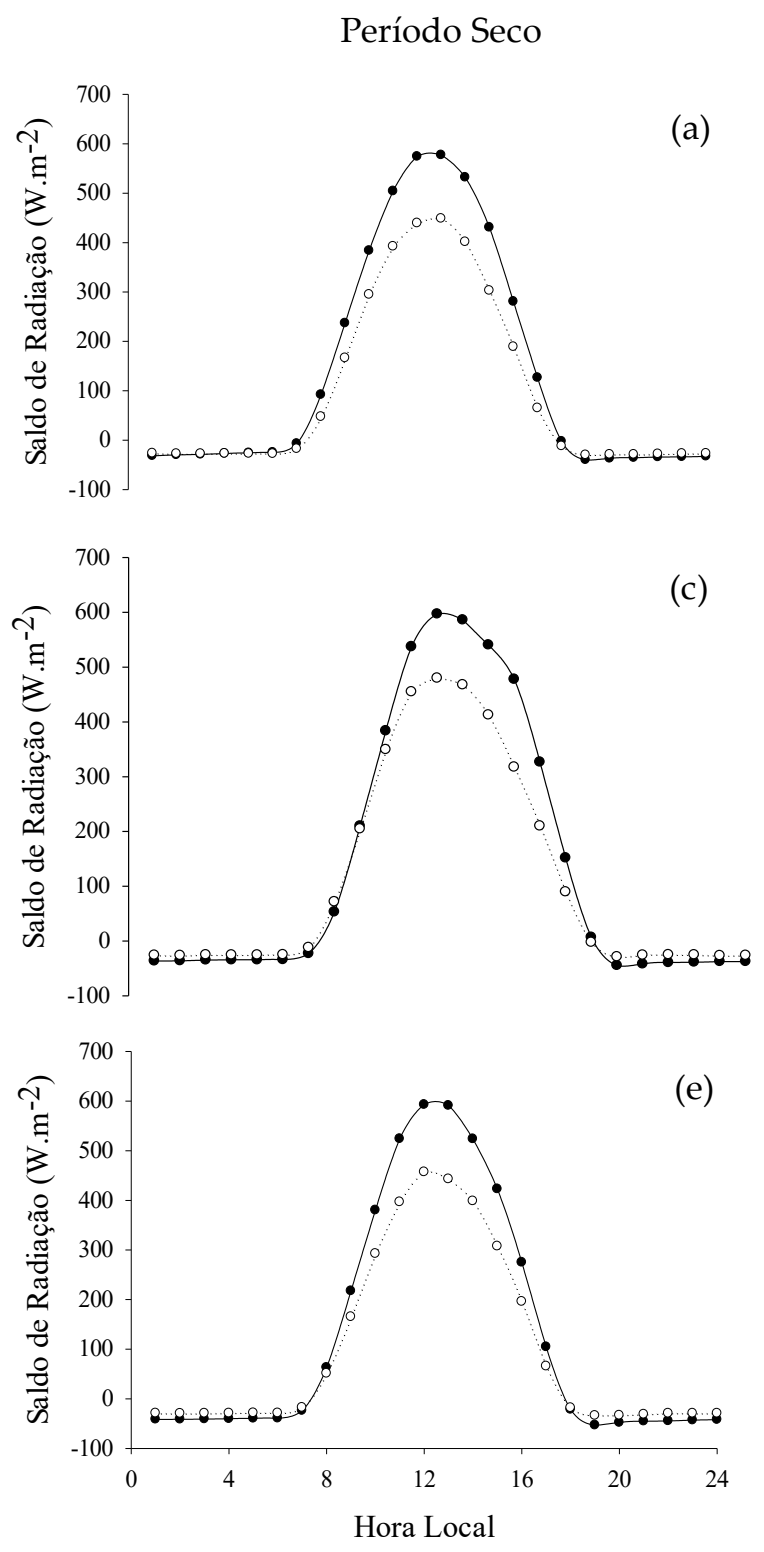

o … FNS

Figura 3: Variação do ciclo diurno médio do saldo de radiação para o período úmido e seco da REBIO Jaru e FNS nos anos de 2005 (a), 2009 (b, c) e 2010 (d, e).

A vegetação da floresta possui menor albedo (devido à disposição e tonalidade das folhas) do que a vegetação da pastagem, fazendo com que na pastagem a refletividade da radiação solar incidente seja maior, contribuindo para uma menor quantidade de energia disponível na 
pastagem. Ao longo do ano, o albedo da pastagem é variável, apresentando maiores valores durante a estação seca, devido à vegetação tornar-se mais seca - com albedo maior - pela menor disponibilidade de água no solo e dessa forma aumentar o albedo na superfície e consequentemente diminuir o saldo de radiação. $\mathrm{O}$ mesmo acontece durante os anos de seca, quando o albedo da pastagem também torna-se maior.

Outra situação que propicia menor saldo radioativo na FNS são os aerossóis atmosféricos provenientes de queimadas. O período seco, bem como, eventos de seca favorecem queimadas em larga escala, o que aumenta a concentração de aerossóis atmosféricos. Dessa forma, a radiação tende a ser absorvida ou espalhada por esses aerossóis, diminuindo a quantidade de radiação solar incidente que alcança à superfície.

Essa situação é evidenciada pelo Instituto Nacional de Pesquisas Espaciais ao relatar um aumento de mais de $200 \%$ no número de focos de queimadas dos anos 2005 e 2010 comparados a 2009 (INPE, 2015).

\section{Conclusões}

Ao comparar os resultados entre os sítios experimentais, a REBIO Jaru apresentou maior umidade específica do ar tanto no período úmido quanto no período seco. Os menores valores de umidade específica foram encontrados nos meses mais secos, bem como, as maiores diferenças entre os sítios.

A área de pastagem apresentou amplitude térmica média superior nos anos com eventos de cheia no período seco, fato não observado na área de floresta.

O saldo de radiação em ambos os sítios foi menor nos anos de 2005 e 2010, provavelmente devido ao expressivo aumento no número de focos de queimadas em relação ao ano de 2009.

Uma pesquisa dessa natureza, além de proporcionar maior entendimento do clima local, permite que as lavouras e atividades agropastoris sejam planejadas levando em consideração situações ocasionadas por eventos extremos, de modo que os prejuízos econômicos e sociais possam ser minimizados.

\section{Agradecimentos}

A Pró-reitoria de Pós-Graduação e Pesquisa PROPesq - da Universidade Federal de Rondônia, do Conselho Nacional de Desenvolvimento Científico e Tecnológico $\mathrm{CNPq}$, por ter possibilitado o desenvolvimento dessa pesquisa e ao LBA pela disponibilização dos dados.

\section{Referências}

Aguiar, R. G., von Randow, C., Priante Filho, N., Manzi, A. O., Aguiar, L. J. G., Cardoso, F. L. (2006). Fluxos de massa e energia em uma floresta tropical no sudoeste da Amazônia. Revista Brasileira de Meteorologia, 21(3b), 248257.

Andrade, N. L. R., Aguiar, R. G., Sanches, L., Alves, E. C. R. F., Nogueira, J. S. (2009). Partição do saldo de radiação em áreas de floresta Amazônica e Floresta de transição AmazôniaCerrado. Revista Brasileira de Meteorologia, 24(3), 346-355.

Andreae, M. O., Artaxo, P., Brandão, C., Carswell, F. E., Ciccioli, P., Costa, A. L., Culf, A. D., Esteves, J. L., Gash, J. H. C., Grace, J., Kabat, P., Lelieveld, J., Malhi, Y., Manzi, A. O., Meixner, F. X., Nobre, A. D., Nobre, C., Ruivo, M. L. P., Silva-Dias, M. A., Stefani, P., Valentini, R., Jouanne, J., Waterloo, M. J. (2002). Biogeochemical cycling of carbon, water, energy, trace gases, and aerosols in Amazonia: The LBAEUSTACH experiments. Journal of Geophysical Research. 107(2), 1-25.

BRASIL (1979). Decreto-lei n. 83.716, de 11 de julho de 1979. Cria, no território Federal de Rondônia, a Reserva Biológica do Jaru. Diário Oficial da União, Brasília, 83, 2. Seção 1.

Culf, A. D, Fisch, G, Malhi, Y, Nobre, C. A. (1997). The influence of the atmospheric boundary layer on carbon dioxide concentrations over a tropical forest. Agricultural and Forest Meteorology, 85, 149-158.

Empresa Brasileira de Pesquisa Agropecuária, Instituto Nacional De Pesquisas Espaciais (2011). Levantamento de Informações de Uso e Cobertura da Terra na Amazônia. Disponível em:

$<$ www.inpe.br/noticias/noticia.php?Cod_Noticia 
$=2654>$ Acesso em: 08 jul. 2014.

Fearnside, P. M. (2009). A vulnerabilidade da floresta amazônica perante as mudanças climáticas. Oecologia Brasiliensis, 13(4), 609-618.

Fearnside, P. M. (2005). Desmatamento na Amazônia brasileira: história, índices e consequências. Megadiversidade, 1(1), 113-123.

Gomes, J. B. (2011). Conversão de florestas tropicais em sistemas pecuários na Amazônia: quais são as implicações no microclima da região? Ji-Paraná-RO: UNIR. Monografia (Graduação em Engenharia Ambiental), Departamento de Engenharia, Universidade Federal de Rondônia.

Hodnett, M. G., Oyama, M. D., Tomasella, J., Marques Filho, A. O. (1996). Comparisons of long-term soil water storage behaviour under pasture and forest in three areas of Amazonia. In: Gash, J. H. C., Nobre, C. A., Roberts, J. M., Victoria, R. L. (Org.). Amazonian Deforestation and Climate. Chichester: John Wiley, 57-77.

Instituto Nacional de Pesquisas Espaciais - INPE (2015). Portal do Monitoramento de Queimadas e Incêndios. Disponível em: < http://www.inpe.br/queimadas>. Acesso em: 07 jul. 2015.

Marengo, J. A. (2009). Impactos de extremos relacionados com o tempo e o clima - Impactos sociais e econômicos. Grupo de Pesquisa em Mudanças Climáticas (GPMC) do Instituto Nacional de Pesquisas Espaciais (INPE). Edição especial.

Marengo, J. A., Tomasella, J., Soares, W. R., Alves, L. M., Nobre, C. A. (2011a). Extreme climatic events in the Amazon basin. Theoretical and Applied Climatology, 107, 73-85.

Marengo, J. A., Tomasella, J., Alves, L. M., Soares, W. R., Rodriguez, D. A. (2011b). The drought of 2010 in the context of historical droughts in the Amazon region. Geophysical Research Letters, 38, 1-5.

Nobre, C. A., Sampaio, G., Salazar, L. (2007). Mudanças climáticas e Amazônia. Ciência e Cultura, 59(3), 21-27.

Nobre, C. A., Seller, P. J., Shukla, J. (1991). Amazonian deforestation and regional climate change. Journal of Climate, 4, 957-988.
Rummel, U., Ammann, C., Gut, A., Meixner, F. X., Andreae, M. O. (2002) Eddy covariance measurements of nitric oxide flux within an Amazonian rain forest. Journal of Geophysical Research, 107, 1-9.

Soares-Filho, B. S., Nepstad, D. C., Curran, L., Cerqueira, G. C., Garcia, R. A., Ramos, C. A., Voll, E., McDonald, A., Lefebvre, P., Schlesinger, P., McGrath, D. (2005). Cenários de desmatamento para a Amazônia. Estudos Avançados, 19(54), 137-152.

Von Randow, C., Manzi, A. O., Kruijt, B., Oliveira, P. J., Zanchi, F. B., Silva, R. L., Hodnett, M. G., Gash, J. H. C., Elbers, J. A., Waterloo, M. J., Cardoso, F. L., Kabat, P. (2004). Comparative measurements and seasonal variations in energy and carbon exchange over forest and pasture in South West Amazonia. Theoretical and Applied Climatology, 78, 5-26.

Webler, A. D., Gomes, J. B., Aguiar, R. G., Andrade, N. L. R., Aguiar, L. J. G. (2013). Mudanças no uso da terra e o particionamento de energia no sudoeste da Amazônia. Revista Brasileira de Engenharia Agrícola e Ambiental, 17(8), 868-876. 